\title{
Crystallization Kinetics in Ge-Sb-Te-Bi and Ge-Sb-Te-In Thin Films
}

\author{
Alexey Sherchenkov ${ }^{1, a}$, Alexey Babich, b , Petr Lazarenko ${ }^{1, ~ c}$, \\ Sergey Timoshenkov ${ }^{1, d}$, Sergey Kozyukhin ${ }^{2, ~ e ~}$ \\ ${ }^{1}$ National Research University of Electronic Technology, Moscow, 124498, Russia \\ ${ }^{2}$ Kurnakov Institute of General and Inorganic Chemistry of the Russian Academy of Sciences, \\ Moscow, 119991, Russia

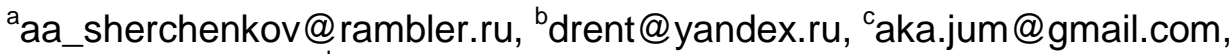 \\ dspt@miee.ru, esergkoz@igic.ras.ru
}

\section{Keywords: Crystallization, Phase change memory, Ge-Sb-Te}

\begin{abstract}
In this work mechanism and kinetics of crystallization for thin films on the basis of $\mathrm{Ge}-\mathrm{Sb}$-Te-Bi and Ge-Sb-Te-In perspective for phase change memory application were investigated. Possible data processing and storage times of the PCM cell were estimated. It was shown that PCM cell based on $\mathrm{Ge}_{2} \mathrm{Sb}_{2} \mathrm{Te}_{5}+0.5 \mathrm{wt}$. \% Bi have minimum data processing and maximum data storage times in comparison with the other investigated materials.
\end{abstract}

\section{Introduction}

Currently phase change memory (PCM) is considered to be one of the most promising candidates for the next generation of non-volatile memory devices [1].

Concept of phase-change memory was proposed by Stanford Ovshinsky in 1968 [2], and demonstrate the possibility of electrical and optical ways of storing information with using of chalcogenide glassy semiconductors $[3,4]$. These lead to the appearance of two main types of PCM memory devices - optical and electrical phase change memories. The work of both types of devices is based on the rapid reversible amorphous-to-crystalline phase transitions, which can be initiated by laser or electrical pulses, respectively. PCM materials in amorphous and crystalline states have very different optical and electrical properties, and are attributed as "zero" and "unit" logical states.

Chalcogenide semiconductors on the quasi-binary line $\mathrm{GeTe}-\mathrm{Sb}_{2} \mathrm{Te}_{3}$ are considered to be most promising programmable materials for application in phase change memories [1].

Three compounds exist on this quasi-binary line: $\mathrm{Ge}_{2} \mathrm{Sb}_{2} \mathrm{Te}_{5}, \mathrm{GeSb}_{2} \mathrm{Te}_{4}$ and $\mathrm{GeSb}_{4} \mathrm{Te}_{7}$. The most promising among them is considered to be $\mathrm{Ge}_{2} \mathrm{Sb}_{2} \mathrm{Te}_{5}$ (GST225) due to the complex of properties (appreciable stability at room temperature, high crystallization rate and good reversibility between amorphous and crystalline phases) [5]. The discovery of GST225 by Yamada et al [5], which lead to the commercial success of CD, DVD, Blu-ray discs.

Noticeable progress was achieved last years in the development of phase change memory. However, improvement of the PCM characteristics is still required. One of the problems is connected with the need of decreasing the data processing time of PCM to the level comparable with that of the random access memory ( 10-50 ns) [1]. For this purpose increasing of crystallization rate for PCM materials is required. The doping can be used for controlling the thermal and increasing crystallization rate. However, most chalcogenide glasses are insensitive to the doping. In addition, the effect of doping on the thermal properties and kinetics of crystallization for GST225 thin films is not well understood. We suppose that Bi and In are promising dopants for the modification of thermal characteristics and crystallization kinetics of GST225, because Bi is isomorphous with $\mathrm{Sb}$, while In and $\mathrm{Sb}$ are in the same period of the Periodic table and have close atomic radiuses. These should provide the replacement mechanism of doping, which will modify binding energies, and consequently thermal properties and crystallization kinetics.

So, the aim of this work was investigation of the crystallization kinetics in Ge-Sb-Te-Bi and Ge-Sb-Te-In thin films. 


\section{Experimental}

The GST225 alloys with different amounts of Bi (from 0 to $7.7 \mathrm{wt} . \%$ ) and In (from 0 to 3 wt. \%) were synthesized with using quenching technique [6]. Thin films were prepared by thermal evaporation of synthesized materials. Substrate temperature during the deposition did not exceed $50^{\circ} \mathrm{C}$.

X-ray diffraction (Rigaku D/MAX, $\mathrm{Cu} \mathrm{Ka} \mathrm{k}=0.15481 \mathrm{~nm}$ ) showed that synthesized materials were polycrystalline and have trigonal modification of $\mathrm{Ge}_{2} \mathrm{Sb}_{2} \mathrm{Te}_{5}$. Introduction of Bi leads to the appearance of trigonal modification of $\mathrm{Bi}_{2} \mathrm{Ge}_{2} \mathrm{Te}_{5}$, which indicates on the replacement of Sb by $\mathrm{Bi}$. As-deposited thin films were amorphous up to $3 \%$ of $\mathrm{Bi}$. Higher concentrations of $\mathrm{Bi}$ lead to the appearance of crystalline phases.

Rutherford backscattering (RBS, $\mathrm{E}_{\mathrm{d}}=1.0$ and $\mathrm{E}_{\alpha}=2.7 \mathrm{MeV}$ at $135^{\circ}$ scattering angle) and Energy Dispersive X-Ray Analysis (EDXRA, Inka-Sight) showed that the compositions of films were close to those of synthesized materials (Tables 1 and 2).

Differential scanning calorimetry (DSC-50, Shimadzu) was used for the investigation of thermal properties. Measurements were carried out at 8 different heating rates (from 1 to $90^{\circ} \mathrm{C} / \mathrm{min}$ ) in a nitrogen flow $(20 \mathrm{ml} / \mathrm{min})$. Thin films were scraped off from c-Si wafers and pressed in $\mathrm{Al}$ pans.

Table 1. Results of composition analysis by RBS for GST 225+Bi thin films.

\begin{tabular}{|l|l|l|l|l|c|}
\hline \multirow{2}{*}{$\begin{array}{l}\text { Initial compounds } \\
\text { (bulk } \\
\text { polycrystalline) }\end{array}$} & \multicolumn{2}{|c|}{ Thin film compositions, at. un., accuracy $\pm 5 \%$} & $\begin{array}{l}\text { Bi content for thin } \\
\text { film (calculation), } \\
\text { wt.\% }\end{array}$ \\
\cline { 2 - 6 } & $\mathrm{Ge}$ & $\mathrm{Sb}+\mathrm{Te}$ & $\mathrm{Bi}$ & Oxygen & \\
\hline GST 225 & 2 & 7 & & & 0.48 \\
\hline GST225+0.5wt.\% $\mathrm{Bi}$ & 2 & $6.80 \pm 0.20$ & $0.024 \pm 0.010$ & $<(0.12 \pm 0.04)$ & 1.07 \\
\hline GST225+1 wt.\% Bi & 2 & $6.86 \pm 0.20$ & $0.053 \pm 0.010$ & $<(0.20 \pm 0.06)$ & 2.75 \\
\hline
\end{tabular}

Table 2 Results of composition analysis by EDXRA for GST 225+In thin films.

\begin{tabular}{|c|c|c|c|c|c|}
\hline \multirow{2}{*}{$\begin{array}{l}\text { Initial compounds } \\
\text { (bulk polycrystalline) }\end{array}$} & \multicolumn{4}{|c|}{ Thin film compositions, at. un. } & \multirow{2}{*}{$\begin{array}{l}\text { Bi content for thin film } \\
\text { (calculation), wt. } \%\end{array}$} \\
\hline & $\mathrm{Ge}$ & $\mathrm{Sb}$ & $\mathrm{Te}$ & In & \\
\hline GST225+0.5 wt. \%In & 0.224 & 0.222 & 0.547 & 0.00463 & 0.45 \\
\hline GST225+1 wt. \%In & 0.197 & 0.263 & 0.527 & 0.00866 & 0.96 \\
\hline GST225+3 wt. \%In & 0.191 & 0.268 & 0.505 & 0.0353 & 3.80 \\
\hline
\end{tabular}

\section{Results and Discussions}

Differential scanning calorimetry of the as-deposited amorphous films revealed a number of heat effects with crystallization peak for all compositions lays in the temperature range of $135-220^{\circ} \mathrm{C}$ (Fig. 1 and 2).

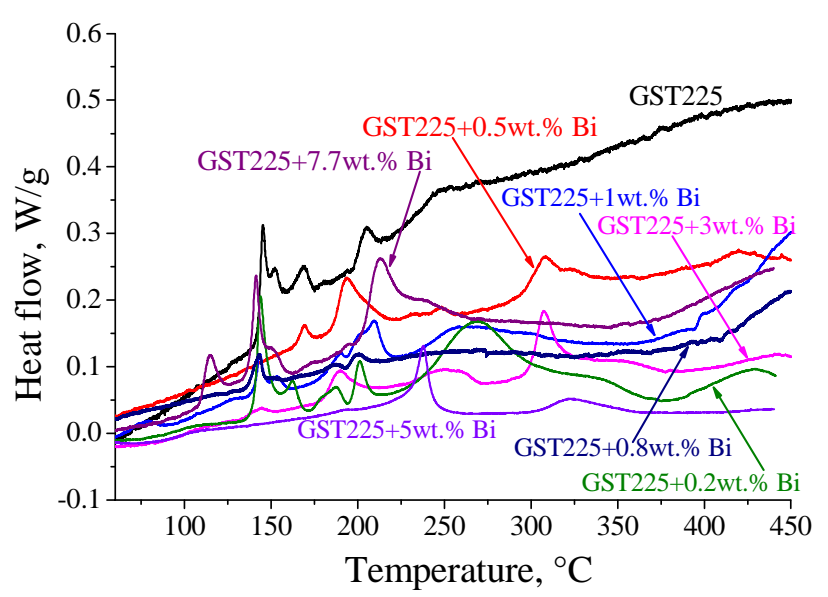

Fig. 1 DSC scans of as-deposited GST225+Bi thin films for heating rate of $10^{\circ} \mathrm{C} / \mathrm{min}$. 


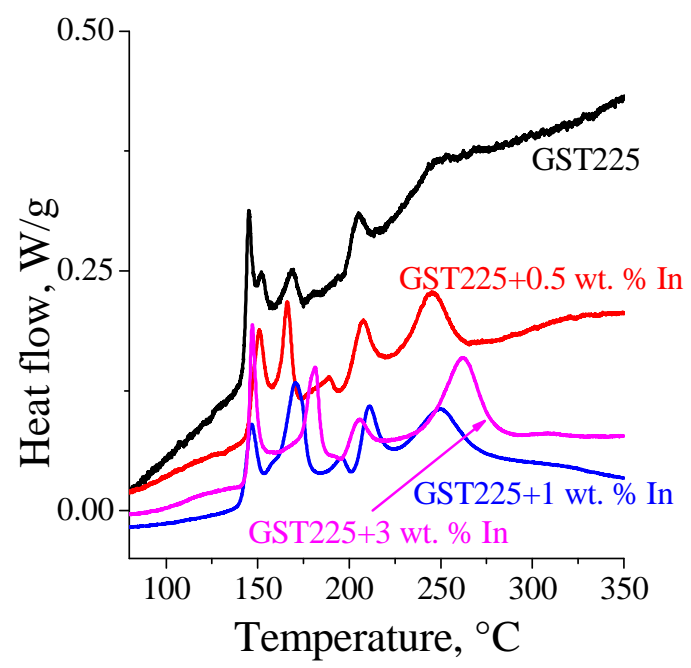

Fig. 2 DSC scans of as-deposited GST225+In thin films for heating rate of $10^{\circ} \mathrm{C} / \mathrm{min}$.

Crystallization temperatures were estimated for all investigated materials, and concentration dependencies of crystallization temperatures are presented in Fig. 3.

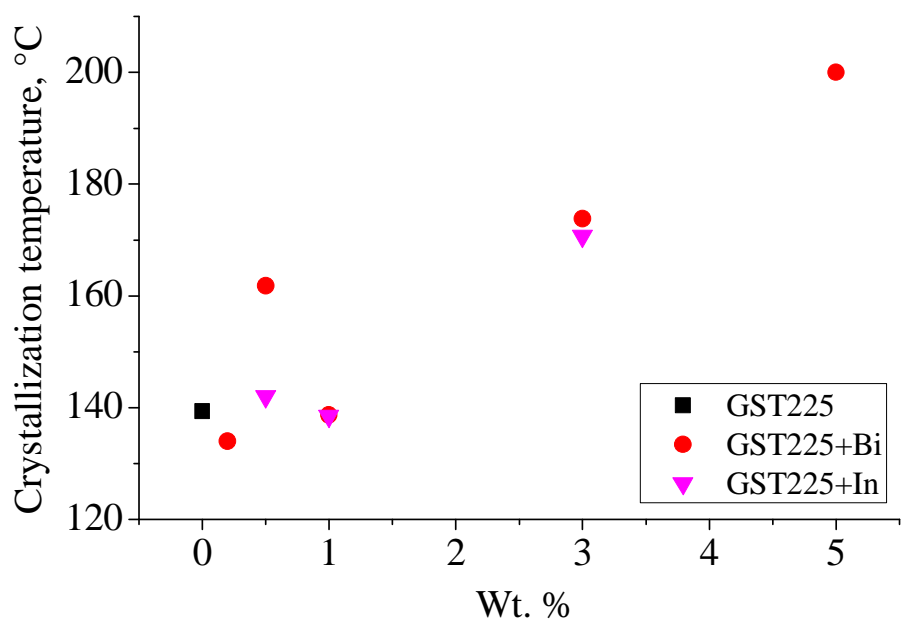

Fig. 3 Concentration dependencies of crystallization temperatures for investigated thin films.

As can be seen from the figure, crystallization temperatures increases with dopant concentration higher than $3 \mathrm{wt}$. \%. At lower concentrations dependencies are not monotonous with the largest deviation for GST225+0.5 wt. \% Bi.

To determine kinetic triplet (effective activation energy of crystallization, pre-exponential factor, crystallization model) for crystallization process of GST thin films we used Isoconversional model-free method of Ozawa-Flynn-Wall (OFW) [7], and Coates-Redfern model-fitting method [7]. Such joint use of model-free and model-fitting methods allowed us to estimate activation energy and pre-exponential factor as functions of conversion, and determine reaction models.

It was found that crystallization process most adequately can be described by the second or third-order reactions, which are characteristic for spontaneous nucleation with further growth without overlapping [8].

Dependencies of the effective activation energy of crystallization on conversion for investigated materials are presented in Fig. 4 and 5. 


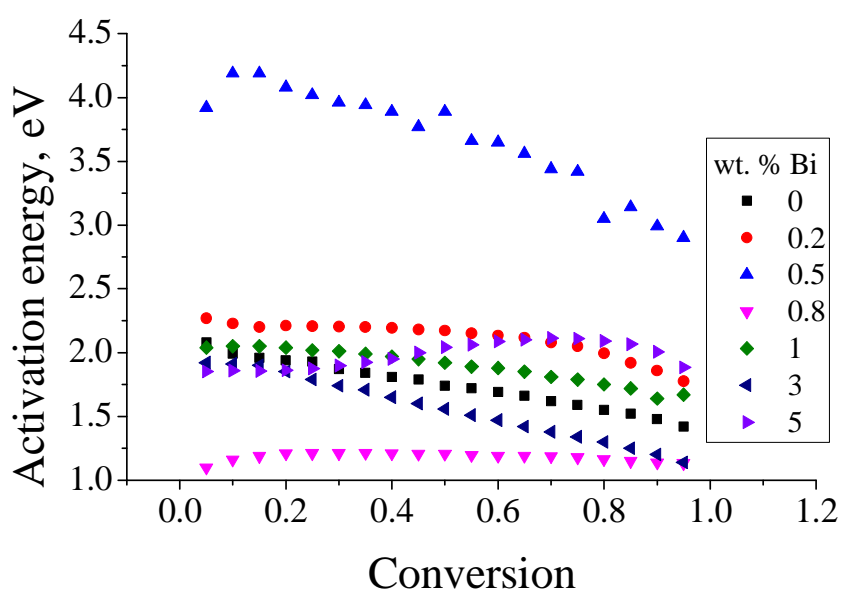

Fig. 4 Effective activation energy of crystallization for compositions with Bi.

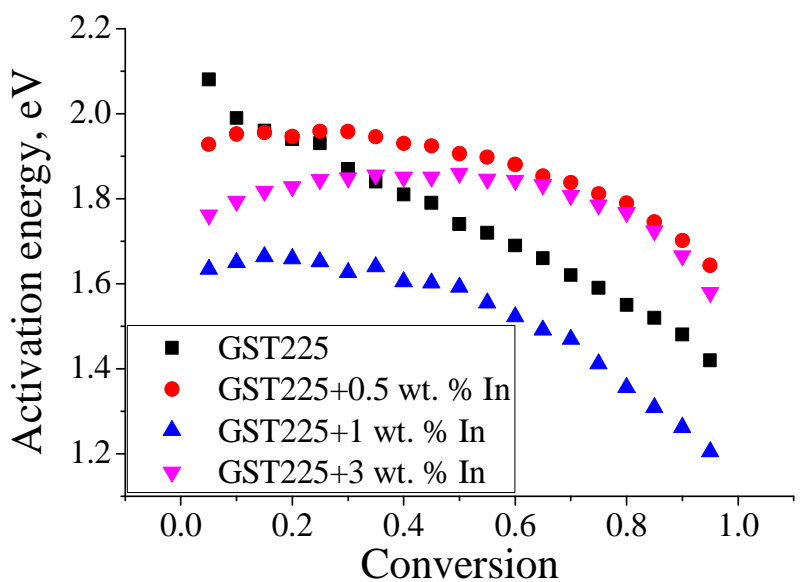

Fig. 5 Effective activation energy of crystallization for compositions with In.

As can be seen from the figures activation energy for undoped GST225 at the beginning of the crystallization is $\sim 2 \mathrm{eV}$, and gradually decreases with the increase of conversion. This result indicates that crystallization of GST films is a complex process consisting at least of two stages. For crystallization process the most possible stages are nucleation and crystal growth. Contribution of the first process gradually decreases, while of the second increases. In this case, activation energy in the initial moment corresponds to the activation energy of nucleation, while in the end - to the activation energy of crystal growth. Similar tendency is observed for GST225+Bi thin films. However, sufficiently higher values of the effective activation energy in comparison with other investigated materials were established for GST225 + 0.5 wt. \% Bi thin films.

For GST225+In slight change of the activation energy at the beginning of crystallization indicates on the enhanced influence of In doping on the nucleation in GST225, which leads to the elongation of the stage controlled by the nucleation.

After the kinetic triplet determination, we used it for the estimation of possible data processing and storage times of the PCM cells on the basis of investigated materials. We supposed that logical "zero" and "unit" states differs by the values of conductivity not less, than by 1-2 orders of magnitude. This allowed us to estimate conversion at which transition between "0" and "1" logical states of the PCM cell occurs by the equation [9]

$$
\alpha=\left(\ln \sigma-\ln \sigma_{a}\right) /\left(n \sigma_{c}-\ln \sigma_{a}\right),
$$

where, $\sigma_{a}$ is the conductivity of a completely amorphous material, $\sigma_{c}$ is the conductivity of a completely crystalline material, and $\sigma$ is the conductivity of the material with the crystalline fraction $\alpha$. 
The calculations showed that the transition between " 0 " and " 1 " occurs at $\alpha=0.45$. By using this value of conversion we estimated possible data processing and storage times of PCM cells as crystallization time of the films on the basis of investigated materials at different temperatures, which can be calculated by the equation [8]:

$$
t=g(\alpha) /\left[A \exp \left(E_{a} /(R T)\right)\right],
$$

where, $g(\alpha)$ is integral form of the found reaction model, A is the pre-exponential factor.

Calculated data processing and storage times for PCM cells on the basis of investigated materials are presented in Fig. 6.

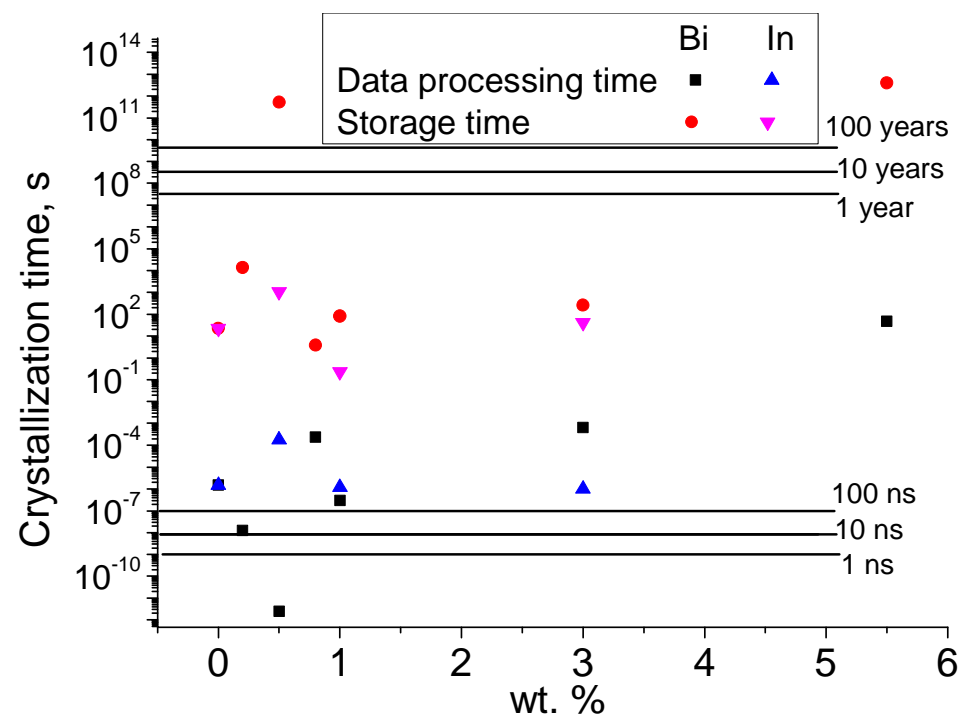

Fig. 6 Data processing and storage times for PCM cells on the basis of investigated materials.

As can be seen from the figure calculations predicts that PCM cells on the basis of $\mathrm{Ge}_{2} \mathrm{Sb}_{2} \mathrm{Te}_{5}+0.5$ mass $\% \mathrm{Bi}$ will have the best data processing and storage times in comparison with the rest compositions. At the crystallization temperature higher than $300{ }^{\circ} \mathrm{C}$ data processing time of the PCM cell on the basis of this material is lower than $\sim 50 \mathrm{~ns}$, which is comparable with the data processing time of the random access memory. In addition, PCM cells on the basis of $\mathrm{Ge}_{2} \mathrm{Sb}_{2} \mathrm{Te}_{5}+$ $0.5 \mathrm{wt}$. \% $\mathrm{Bi}$ has extremely long storage time due to the longest crystallization time at room temperature among all investigated materials.

\section{Conclusion}

Thus, investigations showed that introduction of $\mathrm{Bi}$ and In in $\mathrm{Ge}_{2} \mathrm{Sb}_{2} \mathrm{Te}_{5}$ can affects thermal properties of the material, in particular crystallization kinetics, which is important for the improvement of PCM technology.

It was found that $\mathrm{Ge}_{2} \mathrm{Sb}_{2} \mathrm{Te}_{5}+0.5$ wt. $\% \mathrm{Bi}$ can provide data processing time of the PCM cell comparable with that of the random access memory. In addition, at room temperature this material has extremely long storage time.

\section{Acknowledgements}

This study was supported by Ministry of Education and Science of RF (project ID: RFMEFI57814X0085).

\section{References}

[1] G. W. Burr et al. Phase change memory technology, J. Vacuum Sci. Technol. B. 28(2) (2010) 223-262. 
[2] S. R. Ovshinsky, Reversible Electrical Switching Phenomena in Disordered Structures, Phys. Rev. Lett. 21(20) (1968) 1450-1453.

[3] S. R. Ovshinsky, H. Fritzsche, Reversible structural transformations in amorphous semiconductors for memory and logic, Metall. Trans. 2(3) (1971) 641-645.

[4] J. Feileib et al. Reversible optical effects in amorphous semiconductors, J. Non-Cryst. Solid. 8-10 (1972) 909-916.

[5] N. Yamada et al. High-speed overwritable phase-change optical disc material, Jpn. J. Appl. Phys. 26(26-4) (1987) 61-66.

[6] K. Sergey, S. Alexey, G Ekaterina, K. Vera, V. Alexandr, Thermal effects in Ge-Sb-Te phasechange memory materials during multiple thermal cycling, Phys. Status Solidi, 7(3-4) (2010) 848-851.

[7] K. Sergey, S. Alexey, B. Alexey, L. Petr, N. Huy Phuc, P. Oleg, Peculiarities of Bi doping of Ge-Sb-Te thin films for PCM devices. Can. J. Phys. 92(7/8) (2014) 684-689.

[8] Brown. Handbook of thermal analysis and Calorimetry. Amsterdam: Elsiever Science B.V. 1998.

[9] N. Mehta, A. Kumar, A study of thermal crystallization in glassy $\mathrm{Se}_{80} \mathrm{Te}_{20}$ and $\mathrm{Se}_{80} \mathrm{In}_{20}$ using DSC technique, J. Therm. Anal. Calorim. 83(2) (2006) 669-673. 